\title{
Bivariate Genomic Prediction of Phenotypes by Selecting Epistatic Interactions Across Years Based on Haplotype Blocks and Pruned Sets of SNPS
}

Elaheh Vojgani ( $\sim$ vojgani@gwdg.de)

University of Goettingen, Center for Integrated Breeding Research, Animal Breeding and Genetics Group, Goettingen, Germany https://orcid.org/0000-0003-4375-3531

Torsten Pook

University of Goettingen, Center for integrated Breeding Research, Animal Breeding and Genetics Group, Goettingen

\section{Armin C. Hölker}

Technical University of Munich, School of Life Sciences Weihenstephan, Plant Breeding, Freising

\section{Manfred Mayer}

Technical University of Munich, TUM School of Life Sciences Weihenstephan, Plant Breeding, Freising

\section{Chris-Carolin Schön}

Technical University of Munich, TUM School of Life Sciences Weihenstephan, Plant Breeding, Freising Henner Simianer

University of Goettingen, Center for integrated Breeding Research, Animal Breeding and Genetics Group, Goettingen

\section{Research Article}

Keywords: Epistasis, Bivariate GBLUP, Prediction across years, Genomic correlation, Haplotype blocks

Posted Date: May 20th, 2021

DOI: https://doi.org/10.21203/rs.3.rs-519981/v1

License: (c) (i) This work is licensed under a Creative Commons Attribution 4.0 International License. Read Full License 


\title{
1 Bivariate genomic prediction of phenotypes by selecting epistatic interactions 2 across years based on haplotype blocks and pruned sets of SNPs
}

\author{
3 Elaheh Vojgani ${ }^{1 *}$, Torsten Pook ${ }^{1}$, Armin C. Hölker ${ }^{2}$, Manfred Mayer ${ }^{2}$, Chris-Carolin Schön ${ }^{2}$, \\ Henner Simianer ${ }^{1}$ \\ 51 University of Goettingen, Center for Integrated Breeding Research, Animal Breeding and \\ 6 Genetics Group, Goettingen, Germany; ${ }^{2}$ Plant Breeding, TUM School of Life Sciences \\ 7 Weihenstephan, Technical University of Munich, Freising, Germany
}

\section{Key Massage}

A bivariate epistasis model increases the prediction accuracy similarly for selected subsets of epistatic interactions when utilizing haplotype blocks and pruned sets of SNPs by incorporating phenotypic data across years.

\section{Abstract}

The importance of accurate genomic prediction of phenotypes in plant breeding is undeniable, as higher prediction accuracy can increase selection responses. In this study, we investigated the ability of three models to improve prediction accuracy by including phenotypic information from the last growing season. This was done by considering a single biological trait in two growing seasons (2017 and 2018) as separate traits in a multi-trait model. Thus, bivariate variants of the Genomic Best Linear Unbiased Prediction (GBLUP) as an additive model, Epistatic Random Regression BLUP (ERRBLUP) and selective Epistatic Random Regression BLUP (sERRBLUP) as epistasis models were compared with respect to their prediction accuracies for the second year. The results indicate that bivariate ERRBLUP is almost identical to bivariate GBLUP in prediction accuracy, while bivariate SERRBLUP has the highest prediction accuracy in most cases. The obtained prediction accuracies were similar when utilizing pruned sets of SNPs and haplotype blocks, while utilizing haplotype blocks reduces the computational load significantly compared to utilizing pruned sets of SNPs. The prediction accuracies of bivariate GBLUP, ERRBLUP and sERRBLUP have been assessed across eight phenotypic traits and studied datasets from 471/402 doubled haploid lines in the European maize landrace Kemater Landmais Gelb/Petkuser Ferdinand Rot. We further investigated the genomic correlation, phenotypic correlation and trait heritability as factors affecting the bivariate models' prediction accuracy, with genetic correlation between growing seasons being the most important one. For all three considered model architectures results were far worse when using a univariate version of the model.

\section{Keywords:}

Epistasis, Bivariate GBLUP, Prediction across years, Genomic correlation, Haplotype blocks 
37 Funding

38 This work was funded by German Federal Ministry of Education and Research (BMBF) within the

39 scope of the funding initiative "Plant Breeding Research for the Bioeconomy" (MAZE - "Accessing

40 the genomic and functional diversity of maize to improve quantitative traits"; Funding ID:

41 031B0195)

\section{Conflict of interest}

43 On behalf of all authors, the corresponding author states that there is no conflict of interest.

$44 \quad$ Ethics approval

45 The authors declare that this study complies with the current laws of the countries in which the 46 experiments were performed.

47 Consent to participate

$48 \quad$ Not applicable

49 Consent for publication

50 Not applicable

$51 \quad$ Availability of data and materials

52 All data and material are available through material transfer agreements upon request.

53 Code availability

54 Not applicable

\section{Authors' contributions}

56 EV proposed epistasis models based on haplotype blocks, analyzed the data, wrote the

57 manuscript; TP proposed epistasis relationship matrices; $\mathrm{ACH}, \mathrm{MM}$ and CCS prepared the 58 material; $\mathrm{ACH}$ proposed cross validation strategy in bivariate model; HS proposed the original 59 research question, guided the structure of the research.TP ACM MM CCS HS read, revised and 60 approved the manuscript.

\section{Corresponding author}

62 Correspondence to Elaheh Vojgani

63 Email: vojgani@gwdg.de

64 ORCID: 0000-0003-4375-3531

65 


\section{Acknowledgements}

67 We are thankful to KWS SAAT SE, Misión Biológica de Galicia, Spanish National Research Council 68 (CSIC), Technical University of Munich, and University of Hohenheim for providing the extensive 69 phenotypic evaluation. We are grateful to the German Federal Ministry of Education and 70 Research (BMBF) for the funding of our project within the scope of the funding initiative "Plant 71 Breeding Research for the Bioeconomy" (MAZE - "Accessing the genomic and functional diversity 72 of maize to improve quantitative traits"; Funding ID: 031B0195). 
In plant breeding, genomic prediction has become a regular tool (Bernal-Vasquez et al. 2014; Stich and Ingheland 2018) which enables the optimization of phenotyping costs of breeding programs (Akdemir and Isidro-Sánchez 2019). The importance of genomic prediction of phenotypes is not restricted to plants. Livestock (Daetwyler et al. 2013) and human research (de los Campos et al. 2013) also have been widely developed in this regard. In the context of plant and animal breeding, accurately predicting phenotypic traits is of special importance, since raising all animals and growing all crops to measure their performances requires a considerable amount of money under limited resources (Martini et al. 2016).

Several statistical models have been compared over the last decades in the term of prediction accuracy. In this context, genomic best linear unbiased prediction (GBLUP) (Meuwissen et al. 2001; VanRaden 2007) as an additive linear mixed model has been widely used due to its high robustness, computing speed and superiority in predictive ability to alternative prediction models like Bayesian methods, especially in small reference populations (Da et al. 2014; Rönnegård and Shen 2016; Covarrubias-Pazaran et al. 2018; Wang et al. 2018). Furthermore, the inclusion of genotype $\times$ environment interaction into additive genomic prediction models can result in an increase in prediction accuracy (Hallauer et al. 2010; Bajgain et al. 2020). Such approaches allow borrowing information across environments which potentially leads to higher accuracy in phenotype prediction in multi environment models (Burgueño et al. 2012). In fact, multivariate mixed models have been originally proposed in the context of animal breeding (Henderson and Quaas 1976) with the purpose of modeling the genomic correlation among traits, longitudinal data, and modeling genotype by environment interactions across multiple years or environments (Mrode 2014; Lee and van der Werf 2016; Covarrubias-Pazaran et al. 2018). A multivariate GBLUP model was reported to have higher prediction accuracy than univariate GBLUP (Jia and Jannink 2012) when the genetic correlations were medium (0.6) or high (0.9) (Covarrubias-Pazaran et al. 2018). It was also shown that aggregating the phenotypic data over years to train the model and predict the performance of lines in the following years is a possible approach which can improve prediction accuracy (Auinger et al. 2016; Schrag et al. 2019a).

103 In addition, the inclusion of epistasis, defined as the interaction between loci (Falconer and Mackay 1996; Lynch and Walsh 1998), into the genomic prediction model results in more accurate phenotype prediction (Hu et al. 2011; Wang et al. 2012; Mackay 2014; Martini et al. 2016; Vojgani et al. 2021) due to the considerable contribution of epistasis in genetic variation of quantitative traits (Mackay 2014). In this context, several statistical models have been proposed. Extended genomic best linear unbiased prediction (EG-BLUP, Jiang and Reif 2015) and categorical epistasis (CE, Martini et al. 2017) models are using a marker-based epistatic relationship matrix that is constructed in a highly efficient manner. It has been shown that the CE model is as good as or better than EG-BLUP and does not possess undesirable features of EG-BLUP such as codingdependency (Martini et al. 2017). 
Moreover, it was shown that the accuracy of the epistasis genomic prediction model can be increased in one environment by variable selection in another environment (Martini et al. 2016). In this approach, the full epistasis model was reduced to a model with a subset of the largest epistatic interaction effects, resulting in an increase in predictive ability (Martini et al. 2016), through borrowing information across environments. Vojgani et al. (2021) showed that the prediction accuracy can be increased even further by selecting the interactions with the highest absolute effect sizes / variances in the epistasis model. The resulting higher computational needs were offset by the development of a highly efficient software package "EpiGP" (Vojgani et al. 2019) to perform computations in a bit-wise manner (Schlather 2020). Thus, enabling to conduct such predictions with data sets of practically relevant size across environments in the same year, both with respect to sample size and number of markers (Vojgani et al. 2021). As the number of interactions to account for increases quadratically with the number of included variables, the computational load of methods like EpiGP can quickly go out of control as a model with 600.000 SNPs, as present in high density arrays (Kranis et al. 2013; Unterseer et al. 2014), would result in more than a hundred billion interactions to account for. The most common methods for variable reduction applied here is LD pruning (Purcell et at. 2007), but new linkage-based haplotyping methods (Pook et al. 2019) have recently been proposed to even further reduce the dimensionality of genomic data without much information loss.

The aim of this study is to assess the bivariate genomic prediction models which incorporate epistatic interactions with the target of borrowing information across years to maximize the predictive ability based on both, a pruned set of SNPs and haplotype blocks. Since the accuracy of genomic prediction of phenotypes was shown to be increased by both borrowing information across environments and years (Covarrubias-Pazaran et al. 2018; Schrag et al. 2019b) and inclusion of epistasis into the prediction model (Martini et al. 2016; Vojgani et al. 2020), we combine these two approaches to make the best use of the available information. We further aim to assess the optimum proportion of epistatic interactions to be kept in the model in the variable selection step across years and compare the obtained predictive ability when utilizing pruned sets of SNPs and haplotype blocks. The data used for this purpose were generated in multi-location trials of doubled haploid (DH) lines generated from two European maize landraces 141 in 2017 and 2018.

\section{Materials and Methods}

\section{Data used for analysis}

144 A set of 948 doubled haploid lines of the European maize landraces Kemater Landmais Gelb (KE, 145 Austria, 516 lines) and Petkuser Ferdinand Rot (PE, Germany, 432 lines) were genotyped with the 600 k Affymetrix ${ }^{\circledR}$ Axiom $^{\circledR}$ Maize Array (Unterseer et al. 2014).

147 After quality filtering and imputation, $910 \mathrm{DH}$ lines remained (501 lines in KE and 409 lines in PE) 148 and the panel of markers reduced to 501,124 markers (Hölker et al. 2019). Additionally, loci that 149 were in high level of pairwise linkage disequilibrium (LD) were removed (Calus and Vandenplas 
2018) through linkage disequilibrium based SNP pruning with PLINK v1.07 (Purcell et al. 2007; Chang et al. 2015). LD pruning was done by the parameters of 50, 5 and 2 which considered as the SNPs window size, the number of SNPs at which the SNP window shifts and the variance inflation factor, respectively. This resulted in a data panel containing 25'437 SNPs for KE and 30'212 SNPs for PE (Vojgani et al. 2020). Note that even a panel of 25'000 SNPs results in more than 1 billion SNP interactions to account for. Therefore, in order to have further variable reduction, haplotype blocks as a combination of closely linked markers, which has shown to be an alternative approach for genomic prediction improving the prediction accuracy (Meuwissen et al. 2014; Jiang et al. 2018), have been generated from the full panel of markers with the software HaploBlocker (Pook et al 2019) using default settings. This resulted in a data panel containing 2'972 haplotype blocks in KE and 3'330 haplotype blocks in PE.

Out of 910 genotyped lines only $873 \mathrm{DH}$ lines were phenotyped (471 lines in KE and 402 lines in PE). Einbeck (EIN, Germany), Roggenstein (ROG, Germany), Golada (GOL, Spain) and Tomeza (TOM, Spain) were the four locations that these lines were phenotyped for a series of traits in both 2017 and 2018.

The means, standard deviations, maximum and minimum values of studied phenotypic traits in 2017 and 2018 in each landrace are compared in Table 1 which were derived from the Best Linear Unbiased Estimations (BLUEs) of the genotype mean for each phenotypic trait by Hölker et al. (2019). The comparison of the respective detailed values for each trait in each environment and landrace in 2017 and 2018 are illustrated in the supplementary (Table S1). Vi in phenotypic traits represents the vegetative growth stage when $i$ leaf collars are visible based on the leaf collar method of the corn growth (Abendroth et al. 2011). Early vigour at V3 stage (EV_V3), female flowering (FF) and root lodging ( $R L$ ) were not phenotyped in all four environments for both years. EV_V3 was not phenotyped in EIN in 2018, FF was not phenotyped in GOL in 2017 and RL was not phenotyped in TOM and GOL in both 2017 and 2018.

The number of phenotyped lines per year and environment for trait $\mathrm{PH}$ _ 4 , as the main trait in this study, are summarized in Table 2. For EIN and ROG a higher number of phenotyped lines were generated in 2017. On the contrary, more lines were phenotypes in GOL and TOM in 2018.

\section{Statistical models for phenotype prediction}

We used the bivariate statistical framework as the basis of the genomic prediction models which has been proposed in the recent work by Vojgani et al. (2020). In this regard, GBLUP, ERRBLUP and SERRBLUP as three different methods described in Vojgani et al. (2021) were used for genomic prediction of phenotypes which differ in dispersion matrices representing their covariance structure of the genetic effects. GBLUP as an additive model is based on a genomic relationship matrix calculated according to VanRaden (2008). ERRBLUP (Epistatic Random Regression BLUP) as a full epistasis model is based on all pairwise SNP interactions which generates a new marker matrix considered as a marker combination matrix. The marker combination matrix is a 0,1 matrix indicating the absence (0) or presence (1) of each marker 
combination for each individual. SERRBLUP (selective Epistatic Random Regression BLUP) as a selective epistasis model is based on a selected subset of SNP interactions (Vojgani et al. 2021). Vojgani et al. (2020) proposed estimated effect variances in the training set as the selection criterion of pairwise SNP interactions due to its robustness in predictive ability specifically when only a small proportion of interactions are maintained in the model.

\section{Assessment of genomic prediction models}

GBLUP, ERRBLUP and SERRBLUP models have been assessed via 5-fold cross validation by randomly partitioning the original sample into 5 equal size subsamples in which one subsample was considered as the test set to validate the model, and the remaining 4 subsamples were considered as a joint training set (Erbe et al. 2010). The 5-fold cross validation technique was utilized with 5 replicates through which the Pearson correlation between the predicted genetic values and the observed phenotypes in the test set was considered as the predictive ability in each fold of each replicate, which then was averaged across 25 replicates. In this study, predictive ability was separately assessed for KE and PE for a series of phenotypic traits in four different environments. Besides, we calculated the traits' prediction accuracies by dividing their predictive abilities by the square-root of the respective traits' heritabilities (Dekkers 2007) derived from all environments in both 2017 and 2018 jointly (Table S2).

Univariate GBLUP within 2018 was assessed by training the model in the same year (2018) as the test set was sampled from. However, bivariate GBLUP, ERRBLUP and sERRBLUP were assessed by training the model with both the training set of the target environment in 2018 and the full dataset of the respective environment in 2017. The interaction selection step in bivariate sERRBLUP is done by first using the complete dataset of target environment in 2017 to estimate all pairwise SNP interaction effect variances. Then, an epistatic relationship matrix for all lines is constructed based on the subset of top ranked interaction effect variances, which is finally used to predict phenotypes of the target environment test set in 2018 (Vojgani et al. 2020).

\section{Variance component estimation}

Variance component estimation in univariate GBLUP was done by EMMREML (Akdemir and Godfrey 2015) based on the training set in each run of 5-fold cross validation with 5 replicates. In bivariate models this was done by ASReml-R (Butler et al. 2018) with the approach specified by Vojgani et al. (2020) for pre estimating the variance components from the full dataset to derive the initial values for the variance components in ASReml models in 100 iterations for each combination. If the variance estimation based on the full set did not converge after 100 iterations, then the estimated variance components at the $100^{\text {th }}$ iteration were extracted as initial values of the bivariate model in the cross validation step. Afterwards, the model used these values to re-estimate the variance components based on the training set in each run of 5-fold cross validation in 50 iterations. The estimated variance components in the converged models based on the full set deviated only slightly from the estimated variance components based on the training set (Fig. S1). However, the variance component estimations did not converge in all 
folds of 5-fold cross validation with 5 replicates. In such cases, the initial values were set as the fixed values for the model to predict the breeding values. This approach appears justifiable in the case of non-convergence of the bivariate model, since we have shown in Fig. S2 that the difference in mean predictive ability of all folds and only the converged folds is not critical. This difference can get higher as the number of non-converged folds increases. The number of not converged folds in all studied material is shown in the supplementary (Table S3).

\section{Genomic correlation estimation}

Genomic correlations were estimated from the genetic variances and covariance derived from the ASReml bivariate model based on the full dataset of each location in both 2017 and 2018 .

\section{Results}

\section{Comparison of univariate GBLUP, bivariate GBLUP, bivariate ERRBLUP and bivariate sERRBLUP} based on pruned set of SNPs and haplotype blocks in PH_V4

Our results confirm that bivariate models outperform the univariate models (Vojgani et al. 2020) as illustrated by the comparison in predictive ability of bivariate GBLUP and univariate GBLUP for the trait PH-V4 in both landraces indicating the superiority of bivariate GBLUP to univariate GBLUP in most cases (see Fig. 1 and Fig. 2). Among the bivariate genomic prediction models, the predictive ability obtained from bivariate ERRBLUP is almost identical to bivariate GBLUP. This predictive ability increases in bivariate SERRBLUP and the highest gain in accuracy is generally obtained when the top 10 or 5 percent of pairwise SNP interactions are kept in the model. A too strict selection like using only the top 0.001 percent interactions, results in a decrease in predictive ability (see Fig. 1 and Fig. 2).. The number of interactions maintained in the model for each proportion of interactions are tabulated in the supplementary (Table S4). Robustness of the predictive ability depending on the share of selected markers was higher in PE than KE. Moreover, Fig. 1 and Fig. 2 illustrate the comparison between the predictive abilities obtained from the respective genomic prediction models in KE and PE when utilizing pruned set of SNPs and haplotype blocks. It is shown that the GBLUP, ERRBLUP and sERRBLUP (for the optimum proportions of interactions) predictive abilities are almost identical in both pruned set of SNPs and haplotype blocks. It should be noted that the robustness of sERRBLUP when a very small proportions of interactions maintained in the model is higher when utilizing the pruned set of SNPs compared to haplotype blocks. This should not be surprising as the total number of interactions in the HaploBlocker panel is much smaller, thus, leading to a dataset with an extremely low number of explanatory variables (Table S4). Similar patterns are observed across a series of other traits for bivariate models which are shown in the supplementary (Fig. S3-S16). Additionally, the predictive ability of univariate GBLUP by training the model on the average phenotypic values of both 2017 and 2018, when utilizing pruned set of SNPs, was evaluated for a series of phenotypic traits, which yielded quite similar predictive ability as obtained with univariate GBLUP within year 2018 or worse in some cases (Table S5 (KE) and S6 (PE)). 
The absolute gain in predictive ability from univariate GBLUP to maximum bivariate sERRBLUP, when utilizing pruned set of SNPS, was regressed on the respective SERRBLUP genomic correlation between the two respective environments and across the series of studied traits (Fig. 3). Regression coefficients range between 0.14 and 0.48 and thus show a clear association between the absolute gain in prediction accuracy and the genomic correlation between environments. When combining all traits and environments, this correlation is 0.65 ( $p$-value = 0.00018 ) in KE and 0.69 ( $p$-value $=4.393 e-05$ ) in PE. This correlation is also significant for most of the environments when utilizing haplotype blocks (Fig. S17).

\section{Interplay of GBLUP and sERRBLUP prediction accuracy and genomic correlations}

The genomic correlations across years estimated with GBLUP and sERRBLUP based on pruned set of SNPs for the trait PH_V4 are illustrated in Table 3, indicating that the proportion of interactions in bivariate SERRBLUP which maximized the predictive ability are not necessarily linked to the highest genomic correlation. In contrast, the best sERRBLUP for trait PH_V4 is linked to the lowest genomic correlation in most cases. However, this is not the general pattern observed for a series of other traits and the best SERRBLUP for some traits and environments combinations are linked to the highest genomic correlation (Table S7-S13). In fact, there is a significant correlation between the absolute increase in predictive ability from bivariate GBLUP to maximum bivariate SERRBLUP and the difference between genetic correlations estimated with GBLUP and maximum sERRBLUP in both KE and PE when utilizing pruned set of SNPs (Fig. S18).

\section{Correlation between prediction accuracy and the phenotypic correlation in bivariate models}

There might be some tendency that including phenotypes of the previous year into prediction becomes more efficient when the phenotypic correlation between years is high. In this context, the correlation between the absolute gain in predictive ability from univariate GBLUP to maximum bivariate sERRBLUP and the phenotypic correlation among the years (see Table S14) over all studied traits in all four environments and in both landraces was studied. Fig. 4 demonstrates that the maximum correlation between the absolute gain in the respective predictive ability based on the pruned set of SNPs and the phenotypic correlation is obtained in EIN for KE (0.42) and in ROG for PE (0.62). Across all studied traits and environments, there is a significant correlation of 0.55 in KE ( $p$-value $=0.003)$ and 0.50 in PE ( $p$-value $=0.007)$. This correlation is also significant in most of the environments when utilizing haplotype blocks (Fig. S19).

Relative increase in prediction accuracy across all traits in all environments and landraces with bivariate models

Overall, the percentage of relative increase in prediction accuracy from the bivariate GBLUP to the maximum bivariate SERRBLUP based on pruned set of SNPs in both landraces are illustrated 
in Fig. 5 with the average increase of 7.61 percent in KE and 3.47 percent in PE over all studied traits. Among all traits, the maximum relative increase in prediction accuracy for KE is 22.63 percent which was obtained in EV_V6 in EIN, and for PE is 34.59 percent which was obtained in EV_V4 in EIN. However, Fig. 5 shows some slight decreases in prediction accuracy from bivariate GBLUP to maximum bivariate sERRBLUP for some combinations of traits and environment in both landraces. This is more often observed in PE than KE, where the maximum decrease was found in EV_V6 in TOM for both PE (-3.198 percent) and KE (-2.795 percent). Overall, the average relative increase from bivariate GBLUP to maximum bivariate SERRBLUP was over 3 percent in most cases. The absolute increase in prediction accuracy is also illustrated in the supplementary (Fig. S20) indicating the average increase of 0.046 in KE and 0.015 in PE over all combinations of traits and environments. In addition, the absolute increase in prediction accuracy from the bivariate GBLUP to the maximum bivariate SERRBLUP based on haplotype blocks is shown in the supplementary indicating the average absolute increase of 0.034 in KE and 0.013 in PE (Fig. S21). Overall, the increase in prediction accuracy from bivariate GBLUP to maximum bivariate sERRBLUP is significantly higher in KE than PE in both cases of utilizing pruned set of SNPs and haplotype blocks (Fig. S22).

\section{Discussion}

In this study, bivariate ERRBLUP as a full epistasis model incorporating all pairwise SNP interactions is almost identical to bivariate GBLUP. This was expected, since ERRBLUP incorporates a high number of interactions by which a large number of unimportant variables are introduced into the model (Martini et al. 2016), thus introducing potential 'noise' which can prevent gains in predictive ability. In contrast, bivariate sERRBLUP substantially increases the predictive ability compared to bivariate GBLUP which is only caused by inclusion of relevant pairwise SNP interactions. Note that all bivariate models substantially outperformed univariate GBLUP, as phenotypic data of the respective environment in the previous year was used. ERRBLUP and sERRBLUP models have shown to display similar behaviors in the context of univariate statistical setting in the maize dataset for prediction across environments (Vojgani et al. 2020). ERRBLUP, which had been introduced as categorical epistasis (CE) model by Martini et al. (2017), performs as good as the best EG-BLUP which is EG-BLUP with symmetric marker coding. Similarly, selection of optimum proportions of interactions in EG-BLUP has shown to increase the predictive ability compared to the EG-BLUP which includes all pairwise SNP interactions (Martini et al. 2016) for a wheat dataset (Pérez and de los Campos 2014). Consequently, ERRBLUP and SERRBLUP leads to higher predictive ability than EG-BLUP and reduced EG-BLUP with non-symmetric coded markers, respectively. This was shown by Vojgani et al. (2021) in the wheat dataset (Pérez and de los Campos 2014).

Furthermore, in this study we have found that GBLUP, ERRBLUP and maximum sERRBLUP predictive abilities when utilizing haplotype blocks are very similar to the respective models' predictive abilities when utilizing pruned sets of SNPs. This finding is of high relevance in practice, since it helps to overcome the high computational load of epististatic models. The required 
computational time for sERRBLUP based on 3'330 haplotype blocks indicating 5'546'115 interactions was 9 minutes out of which 4 minutes were needed to estimate the pairwise SNP interaction effect variances and 5 minutes were needed to generate the sERRBLUP relationship matrix for a selected proportion of interactions by utilizing the R-package miraculix with 15 cores on a server cluster with Intel E5-2650 (2X12 core 2.2GHz) processors in the released EpiGP Rpackage (Vojgani et al. 2019). As the computing time is increasing approximately quadratically in the number of included markers, the computing time for the respective SNP-based model with 30'212 SNPs took 90 times as long (Vojgani et al. 2020), while it resulted in similar predictive abilities with the absolute difference being less than 0.01 in most cases across all traits in all environments and both landraces (Fig. S23). Although this difference in predictive abilities is statistically significant based on paired t-test, it is not of practical relevance.

Although, sEERBLUP is a method that is using multiple environments, it is not a GxE model (de Leon et al. 2016) in the traditional sense. While GxE models typically assign effect to combination of specific genotypes depending on the environment, the second environment in SEERBLUP is "only" used to detect which markers affect a given trait and use the information to put more focus on these marker in the actual prediction step. The estimation of marker effects itself is then executed only based on the environment itself (or in the case of the bivariate model with some contributions from the second environment but still not in the sense of a traditional GxE model). As a different model is used for each environment, sEERBLUP will of course still assign different marker effects in different environments. As the set of selected marker interactions will however be different between different models, a direct comparison of the effects assigned to specific marker interactions is not statistically sound. Similar to most GxE models (Shin and Lee 2020), the computational load of sEERBLUP using markers is extremely high. However, our suggested use of haplotype blocks massively reduced this problem, while the predictive ability is almost as good as SERRBLUP based on pruned set of SNPS.

It was shown that multivariate GBLUP is superior in predictive ability compared to univariate GBLUP with of medium $(\sim 0.6)$ to high $(\sim 0.9)$ genomic correlations, and that low genomic correlations results in no increase in multivariate GBLUP compared to univariate GBLUP (Covarrubias-Pazaran et al. 2018). Calus et al. (2011) also found an increase of 3 to 14 percent in predictive ability of multi-trait SNP-based models in a simulation study when genetic correlations ranged from 0.25 to 0.75 . In our study, we also found a significant correlation between the absolute gain in prediction accuracy from univariate GBLUP to maximum bivariate sERRBLUP and the respective genomic correlation based on both pruned sets of SNPs $\left(r_{K E}=0.65, r_{P E}=0.69\right)$ and haplotype blocks $\left(r_{K E}=0.47, r_{P E}=0.49\right)$ across all traits and environments combinations.

Moreover, Martini et al. (2016) showed that the predictive ability in one environment can be increased by variable selection in the other environment under the assumption of positive phenotypic correlation between environments. It was shown in a wheat dataset (Pérez and de los Campos 2014), where environments 2 and 3 had the highest phenotypic correlation (0.661), that the predictive ability for phenotype prediction in environment 2 was maximized by variable 
selection in environment 3 and vice versa (Martini et al. 2016). Therefore, the increase in prediction accuracy is expected to be influenced by the phenotypic correlations between the environments or between the years in the same environment in bivariate models. In our study, although 2017 and 2018 were climatically quite different, since 2018 suffered from a major heat stress compared to 2017 (Table 1), we see a significant correlation between the absolute gain in predictive ability from univariate GBLUP to maximum predictive ability of bivariate sERRBLUP and the phenotypic correlation between years in each environment based on both pruned sets of SNPs $\left(r_{K E}=0.55, r_{P E}=0.50\right)$ and haplotype blocks $\left(r_{K E}=0.55, r_{P E}=0.56\right)$.

In addition to the genomic and phenotypic correlations between the years, the trait heritability is another factor which is expected to be influential for such an increase in bivariate sERRBLUP predictive ability as well. Therefore, the traits with lower heritability are expected to obtain less gain in SERRBLUP predictive ability than the traits with higher heritability. This was confirmed in our study, as traits with low heritability (e.g. 0.59 for RL in PE) showed only a small increase in prediction accuracy from univariate GBLUP to maximum bivariate sERRBLUP. However, not all traits with higher heritabilities did necessarily show a higher gain in predictive ability for all traits. It should be noted that the trait heritabilities were calculated on an entry-mean basis (Hallauer et al. 2010) within each KE and PE landraces by Hölker et al. (2019) over all four environments in both years 2017 and 2018 jointly. The trait heritabilities obtained only from 2017 are significantly higher than the trait heritabilities obtained only from 2018 in both KE and PE based on a paired t-test (Table S2). This also results in an increase in predictive ability from univariate GBLUP to maximum bivariate SERRBLUP in KE and PE, since multi-trait models have the potential of increasing the predictive ability when traits with low heritability are joined with traits with higher heritability, given they are genomically correlated (Thompson and Meyer 1986).

It should be noted that the increase in predictive ability from univariate GBLUP to maximum bivariate SERRBLUP is caused by both borrowing information across years and capitalizing on epistasis, while the increase in predictive ability from bivariate GBLUP to maximum bivariate sERRBLUP is caused by accounting for epistasis alone. Overall, the traits behave differently among different environments and landraces due to their genomic correlations, phenotypic correlations and heritabilities. To shed light on this, the maximum increase in prediction accuracy from bivariate GBLUP to bivariate SERRBLUP based on pruned set of SNPs in KE was observed for the trait EV_V6 (0.112) in EIN where the corresponding SERRBLUP genomic correlation (0.809) is higher than the GBLUP genomic correlation (0.768). This trait has a high heritability $(0.90)$ and high phenotypic correlation (0.551) as well. In contrast, the respective prediction accuracy decreases (-0.018) for EV_V6 in TOM for KE indicating the lower sERRBLUP genomic correlation (0.458) than GBLUP genomic correlation (0.703) and the particularly low phenotypic correlation (0.383). It should be noted that the phenotypic correlation does not play a major role for the increase in prediction accuracy from bivariate GBLUP to bivariate SERRBLUP, since both models are bivariate and benefit from the same phenotypic correlations. Therefore, EV_V6 obtaining the maximum and minimum increase in the respective prediction accuracy for KE indicates the significant role of genomic correlation among the possible causes. In general, bivariate sERRBLUP 
417 improves the prediction accuracy compared to bivariate GBLUP more in KE than PE which is 418 potentially due to significantly higher SERRBLUP genomic correlation and heritability in KE 419 compared to $P E$, based on paired t-test.

420 Overall, our results indicate that incorporating a suitable subset of epistatic interactions besides 421 utilizing information across years can substantially increase the predictive ability. The amount of 422 this increase is affected by the genomic and phenotypic correlations between the years and the 423 heritability of the phenotypic trait. Moreover, utilizing haplotype blocks instead of pruned sets 424 of SNPs in epistasis model is proposed, since the obtained predictive abilities of the best epistasis 425 model are quite similar, while the required computational time when utilizing haplotype blocks 426 is significantly lower than the required computational time when utilizing pruned set of SNPs. 427 Therefore, this computationally efficient approach is potentially beneficial for genomic 428 prediction of phenotypes under the assumption of sufficient genomic and phenotypic correlation 429 between years for highly heritable traits. This may allow to reduce the number of lines which 430 have to be phenotyped over several years and thus reduce phenotyping costs which and thus be 431 of high interest in practical plant breeding. 
Fig. 1 Predictive ability for univariate GBLUP within 2018 (orange and red dashed horizontal line), bivariate GBLUP (green and blue dashed horizontal line), bivariate ERRBLUP (open circle) and bivariate SERRBLUP (filled circles and solid line) for trait PH-V4 in KE based on Pruned set of SNPS (left) and haplotype blocks (right). In each plot, the sERRBLUP maximum indicates the maximum predictive ability obtained from bivariate sERRBLUP.

Fig. 2 Predictive ability for univariate GBLUP within 2018 (orange and red dashed horizontal line), bivariate GBLUP (green and blue dashed horizontal line), bivariate ERRBLUP (open circle) and bivariate SERRBLUP (filled circles and solid line) for trait PH-V4 in PE based on pruned sets of SNPS (left) and haplotype blocks (right). In each plot, the sERRBLUP maximum indicates the maximum predictive ability obtained from bivariate SERRBLUP.

Fig. 3 Regression of the absolute increase in predictive ability from univariate GBLUP to maximum bivariate SERRBLUP on the respective SERRBLUP genomic correlation between 2017 and 2018 in KE (left) and in PE (right) for all studied traits. In each panel, the overall linear regression line (gray solid line) with the regression coefficient $(\boldsymbol{b})$ and R-squared $\left(\boldsymbol{R}^{2}\right)$ are shown.

Fig. 4 Regression of the absolute increase in predictive ability from univariate GBLUP to maximum bivariate SERRBLUP on the phenotypic correlation between 2017 and 2018 in KE (left) and in PE (right) for all studied traits. In each panel, the overall linear regression line (gray solid line) with the regression coefficient $(\boldsymbol{b})$ and R-squared $\left(\boldsymbol{R}^{2}\right)$ are shown.

Fig. 5 Percentage of change in prediction accuracy from bivariate GBLUP to the maximum prediction accuracy of bivariate SERRBLUP based on pruned set of SNPs in KE (left side plot) and in PE (right side plot). The average percentage of change in prediction accuracy for each trait and environment is displayed in all rows and columns, respectively.

457 Table 1 Phenotypic trait description and the mean, minimum, maximum and standard deviation 458 of the BLUEs for each phenotypic trait in KE and PE landraces in the years 2017 and 2018.

459 Table 2 Number of KE and PE lines phenotyped in each location for the years 2017 (blue numbers) 460 and 2018 (red numbers) for trait $\mathrm{PH}$ _V4.

461 Table 3 Genomic correlation between 2017 and 2018 in each environment for trait PH_V4 for KE 462 (blue numbers) and PE (red numbers). The blue and red bold numbers with stars indicate which 463 proportion of interactions in bivariate SERRBLUP maximized the predictive ability based on 464 pruned set of SNPs in each environment for KE and PE, respectively. 


\section{References}

466 Abendroth LJ, Elmore RW, Boyer MJ, and Marlay SK (2011) Corn Growth and Development. 467 PMR 1009. Iowa State University of Science and Technology, Cooperative Extension Service, 468 Ames, lowa.

469 Akdemir D and Godfrey OU (2015) EMMREML: Fitting Mixed Models with Known Covariance Structures. Available at: https://cran.r-project.org/package=EMMREML

Akdemir D and Isidro-Sánchez J (2019) Design of training populations for selective phenotyping

472 in genomic prediction. Scientific Reports 9(1446).

473 https://doi.org/https://doi.org/10.1038/s41598-018-38081-6

474 Auinger H-J, Schönleben M, Lehermeier C, Schmidt M, Korzun V, Geiger HH, Piepho H-P, 475 Gordillo A, Wilde P, Bauer E, and Schön C-C (2016) Model training across multiple breeding cycles significantly improves genomic prediction accuracy in rye (Secale cereale L.). Theoretical and Applied Genetics 129(11): 2043-2053. https://doi.org/10.1007/s00122-016-2756-5

478

479

480

481

482

483

484 485

486

487

488

489 490

491

492

493

494

495

496

497

498

499

500

501
Bajgain P, Zhang X, and Anderson JA (2020) Dominance and GxE interaction effects improve genomic prediction and genetic gain in intermediate wheatgrass (Thinopyrum intermedium). The Plant Genome. John Wiley \& Sons, Ltd 13(1): e20012.

https://doi.org/https://doi.org/10.1002/tpg2.20012

Bernal-Vasquez A-M, Möhring J, Schmidt M, Schönleben M, Schön C-C, and Piepho H-P (2014) The importance of phenotypic data analysis for genomic prediction - a case study comparing different spatial models in rye. BMC Genomics 15(1): 646. https://doi.org/10.1186/1471-2164$15-646$

Burgueño J, Campos G de los, Weigel K, and Crossa J (2012) Genomic Prediction of Breeding Values when Modeling Genotype $\times$ Environment Interaction using Pedigree and Dense Molecular Markers. Crop Science 52(2): 707-719. https://doi.org/10.2135/cropsci2011.06.0299

Butler DG, Cullis BR, Gilmour AR, Gogel BJ, and Thompson R (2018) ASReml-R Reference Manual Version 4. VSN International Ltd., Hemel Hempstead

Calus MPL and Vandenplas J (2018) SNPrune: an efficient algorithm to prune large SNP array and sequence datasets based on high linkage disequilibrium. Genetics Selection Evolution 50(1): 34. https://doi.org/10.1186/s12711-018-0404-z

Calus MPL and Veerkamp RF (2011) Accuracy of multi-trait genomic selection using different methods. Genetics Selection Evolution 43(1): 26. https://doi.org/10.1186/1297-9686-43-26

Chang CC, Chow CC, Tellier LC, Vattikuti S, Purcell SM, and Lee JJ (2015) Second-generation PLINK: rising to the challenge of larger and richer datasets. Gigascience 4(7). https://doi.org/10.1186/s13742-015-0047-8

Covarrubias-Pazaran G, Schlautman B, Diaz-Garcia L, Grygleski E, Polashock J, Johnson-Cicalese J, Vorsa N, Iorizzo M, and Zalapa J (2018) Multivariate GBLUP Improves Accuracy of Genomic Selection for Yield and Fruit Weight in Biparental Populations of Vaccinium macrocarpon Ait. 
Frontiers in Plant Science 9(1310). https://doi.org/10.3389/fpls.2018.01310

Da Y, Wang C, Wang S, and Hu G (2014) Mixed Model Methods for Genomic Prediction and Variance Component Estimation of Additive and Dominance Effects Using SNP Markers. PLOS ONE 9(1). https://doi.org/10.1371/journal.pone.0087666

Daetwyler HD, Calus MPL, Pong-Wong R, Campos G de los, and Hickey JM (2013) Genomic Prediction in Animals and Plants: Simulation of Data, Validation, Reporting, and Benchmarking. Genetics 193: 347-365. https://doi.org/10.1534/genetics.112.147983

Dekkers JCM (2007) Prediction of response to marker-assisted and genomic selection using selection index theory. Journal of Animal Breeding and Genetics 124: 331-341. https://doi.org/10.1111/j.1439-0388.2007.00701.x

Erbe M, Pimentel E, Sharifi AR, and Simianer H (2010) Assessment of cross-validation strategies for genomic prediction in cattle. 9th World Congress of Genetics Applied to Livestock Production 129-132

Falconer DS and Mackay TFC (1996) Introduction to Quantitative Genetics. Longman. Essex Engl. Hallauer AR, Carena MJ, and Miranda Filho JB (2010) Quantitative genetics in maize breeding. Springer. Berlin

Henderson CR and Quaas RL (1976) Multiple Trait Evaluation Using Relatives' Records. Journal of Animal Science 43(6): 1188-1197. https://doi.org/10.2527/jas1976.4361188x

Hölker AC, Mayer M, Presterl T, Bolduan T, Bauer E, Ordas B, Brauner PC, Ouzunova M, Melchinger AE, and Schön C-C (2019) European maize landraces made accessible for plant breeding and genome-based studies. Theoretical and Applied Genetics 132(12): 3333-3345. https://doi.org/10.1007/s00122-019-03428-8

Hu Z, Li Y, Song X, Han Y, Cai X, Xu S, and Li W (2011) Genomic value prediction for quantitative traits under the epistatic model. BMC Genet 12(15).

https://doi.org/https://doi.org/10.1186/1471-2156-12-15

Jia Y and Jannink J-L (2012) Multiple-Trait Genomic Selection Methods Increase Genetic Value Prediction Accuracy. Genetics 192(4): 1513 LP - 1522.

https://doi.org/10.1534/genetics.112.144246

Jiang Y and Reif JC (2015) Modeling Epistasis in Genomic Selection. Genetics 201(2): 759-768. https://doi.org/10.1534/genetics.115.177907

Jiang Y, Schmidt RH, and Reif JC (2018) Haplotype-Based Genome-Wide Prediction Models Exploit Local Epistatic Interactions Among Markers. G3: Genes/Genomes/Genetics 8(5): 1687 LP - 1699. https://doi.org/10.1534/g3.117.300548

Kranis A, Gheyas AA, Boschiero C, Turner F, Yu L, Smith S, Talbot R, Pirani A, Brew F, Kaiser P, Hocking PM, Fife M, Salmon N, Fulton J, Strom TM, Haberer G, Weigend S, Preisinger R, Gholami M, Qanbari S, Simianer H, Watson KA, Woolliams JA, and Burt DW (2013) Development of a high density 600K SNP genotyping array for chicken. BMC Genomics 14(1): 
59. https://doi.org/10.1186/1471-2164-14-59

Lee SH and van der Werf JHJ (2016) MTG2: an efficient algorithm for multivariate linear mixed model analysis based on genomic information. Bioinformatics 32(9): 1420-1422. https://doi.org/10.1093/bioinformatics/btw012

de Leon N, Jannink J-L, Edwards JW, and Kaeppler SM (2016) Introduction to a Special Issue on Genotype by Environment Interaction. Crop Science. John Wiley \& Sons, Ltd 56(5): 2081-2089. https://doi.org/10.2135/cropsci2016.07.0002in

de los Campos G, Vazquez Al, Fernando R, Klimentidis YC, and Sorensen D (2013) Prediction of Complex Human Traits Using the Genomic Best Linear Unbiased Predictor. PLoS Genetics 9(7). https://doi.org/10.1371/journal.pgen.1003608

Lynch M and Walsh B (1998) Genetics and Analysis of Quantitative Traits. Sinauer Associates Mackay TFC (2014) Epistasis and Quantitative Traits: Using Model Organisms to Study GeneGene Interactions. Nat Rev Genet. 15(1): 22-33. https://doi.org/10.1038/nrg3627

Martini JWR, Wimmer V, Erbe M, and Simianer H (2016) Epistasis and covariance: how gene interaction translates into genomic relationship. Theoretical and Applied Genetics 129(5): 963976. https://doi.org/10.1007/s00122-016-2675-5

Martini JWR, Gao N, Cardoso DF, Wimmer V, Erbe M, Cantet RJC, and Henner S (2017) Genomic prediction with epistasis models: on the marker-coding-dependent performance of the extended GBLUP and properties of the categorical epistasis model (CE). BMC Bioinformatics 18(3). https://doi.org/10.1186/s12859-016-1439-1

Meuwissen THE, Odegard J, Andersen-Ranberg I, and Grindflek E (2014) On the distance of genetic relationships and the accuracy of genomic prediction in pig breeding. Genetics Selection Evolution 46(1): 49. https://doi.org/10.1186/1297-9686-46-49

Meuwissen THE, Hayes BJ, and Goddard ME (2001) Prediction of total genetic value using genome-wide dense marker maps. Genetics 157(4): 1819-1829

Mrode RA (2014) Linear Models for the Prediction of Animal Breeding Values. CABI. https://doi.org/10.1079/9781780643915.0000

Pérez P and de los Campos G (2014) Genome-wide regression and prediction with the BGLR statistical package. Genetics. 2014/07/09. Genetics Society of America 198(2): 483-495. https://doi.org/10.1534/genetics.114.164442

Purcell S, Neale B, Todd-Brown K, Thomas L, Ferreira MAR, Bender D, Maller J, Sklar P, Bakker PIW de, Daly MJ, and Sham PC (2007) PLINK: A Tool Set for Whole-Genome Association and Population-Based Linkage Analyses. American Journal of Human Genetics 81(3): 559-575. https://doi.org/10.1086/519795

Rönnegård L and Shen X (2016) Genomic prediction and estimation of marker interaction effects. bioRxiv 38935. https://doi.org/10.1101/038935 
Schlather M (2020) Efficient Calculation of the Genomic Relationship Matrix. bioRxiv. https://doi.org/10.1101/2020.01.12.903146

Schrag TA, Schipprack W, and Melchinger AE (2019a) Across-years prediction of hybrid performance in maize using genomics. Theoretical and Applied Genetics. Springer Verlag 132(4): 933-946. https://doi.org/10.1007/s00122-018-3249-5

Schrag TA, Schipprack W, and Melchinger AE (2019b) Across-years prediction of hybrid performance in maize using genomics. Theoretical and Applied Genetics 132: 933-946

Shin J and Lee SH (2020) GxEsum: genotype-by-environment interaction model based on summary statistics. bioRxiv 2020.05.31.122549. https://doi.org/10.1101/2020.05.31.122549

Stich B and Ingheland D Van (2018) Prospects and Potential Uses of Genomic Prediction of Key Performance Traits in Tetraploid Potato. Frontiers in Plant Science 9(159). https://doi.org/10.3389/fpls.2018.00159

Thompson R and Meyer K (1986) A review of theoretical aspects in the estimation of breeding values for multi-trait selection. Livestock Production Science 15(4): 299-313.

https://doi.org/https://doi.org/10.1016/0301-6226(86)90071-0

Unterseer S, Bauer E, Haberer G, Seidel M, Knaak C, Ouzunova M, Meitinger T, Strom TM, Fries R, Pausch H, Bertani C, Davassi A, Mayer KF, and Schön C-C (2014) A powerful tool for genome analysis in maize: 584 development and evaluation of the high density $600 \mathrm{k}$ SNP genotyping array. BMC Genomics 15(823). https://doi.org/10.1186/1471-2164-15-823

VanRaden P (2007) Efficient estimation of breeding values from dense genomic data. Journal of Dairy Science 90: $374-375$

VanRaden P (2008) Efficient methods to compute genomic predictions. Journal of Dairy Science 91(11): 4414-4423. https://doi.org/10.3168/jds.2007-0980

Vojgani E, Pook T, Martini JWR, Hoelker AC, Mayer M, Schoen C-C, and Simianer H (2020) Accounting for epistasis improves genomic prediction of phenotypes with univariate and bivariate models across environments. bioRxiv 2020.10.08.331074. https://doi.org/10.1101/2020.10.08.331074

Vojgani E, Pook T, and Simianer H (2019) EpiGP: Epistatic relationship matrix based genomic prediction of phenotypes. Available at: https://github.com/evojgani/EpiGP

Vojgani E, Pook T, and Simianer H (2021) Phenotype Prediction under Epistasis. in KC, W. (ed.) Epistasis: Methods and Protocols. Springer. https://doi.org/10.1007/978-1-0716-0947-7_8

Wang D, El-Basyoni IS, Baenziger PS, Crossa J, Eskridge KM, and Dweikat I (2012) Prediction of genetic values of quantitative traits with epistatic effects in plant breeding populations. Heredity 109(5): 313-319. https://doi.org/10.1038/hdy.2012.44

Wang J, Zhou Z, Zhang Zhe, Li H, Liu D, Zhang Q, Bradbury PJ, Buckler ES, and Zhang Zhiwu (2018) Expanding the BLUP alphabet for genomic prediction adaptable to the genetic architectures of complex traits. Heredity 121(6): 648-662. https://doi.org/10.1038/s41437-018- 
$612 \quad 0075-0$

613 

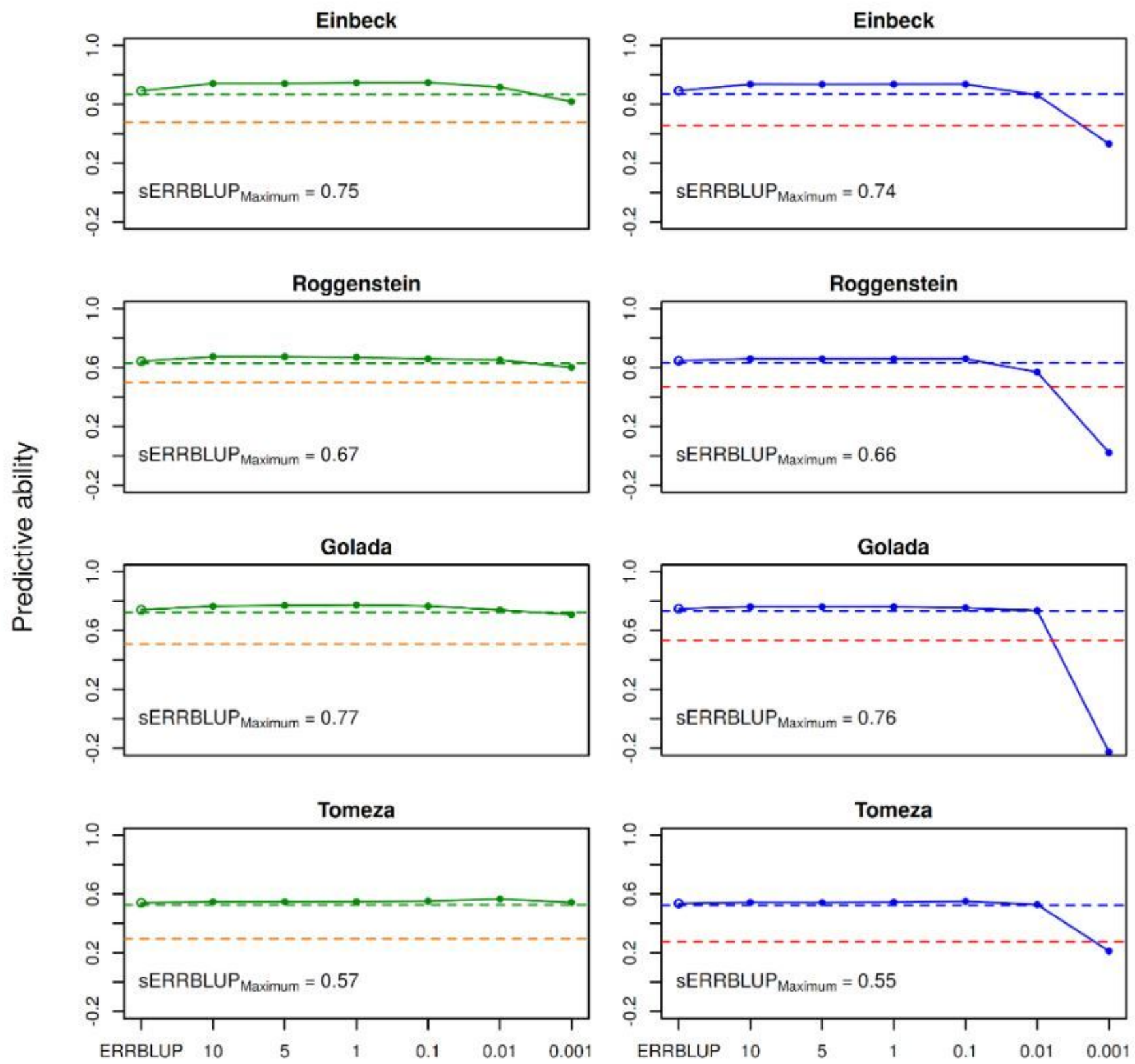

Percentage of interactions maintained in the model

\section{Figure 1}

Predictive ability for univariate GBLUP within 2018 (orange and red dashed horizontal line), bivariate GBLUP (green and blue dashed horizontal line), bivariate ERRBLUP (open circle) and bivariate sERRBLUP (filled circles and solid line) for trait PH-V4 in KE based on Pruned set of SNPs (left) and haplotype blocks (right). In each plot, the sERRBLUP maximum indicates the maximum predictive ability obtained from bivariate sERRBLUP. 
Einbeck

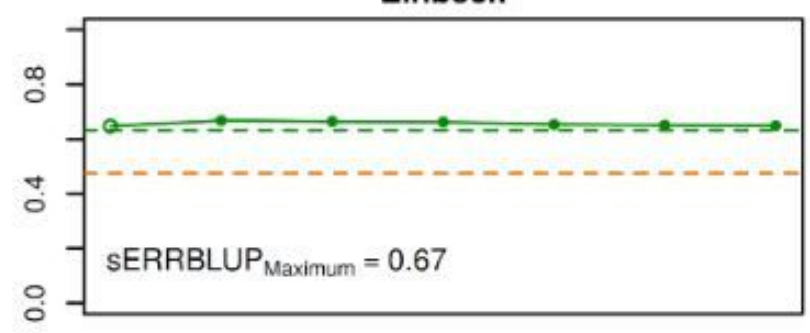

Roggenstein

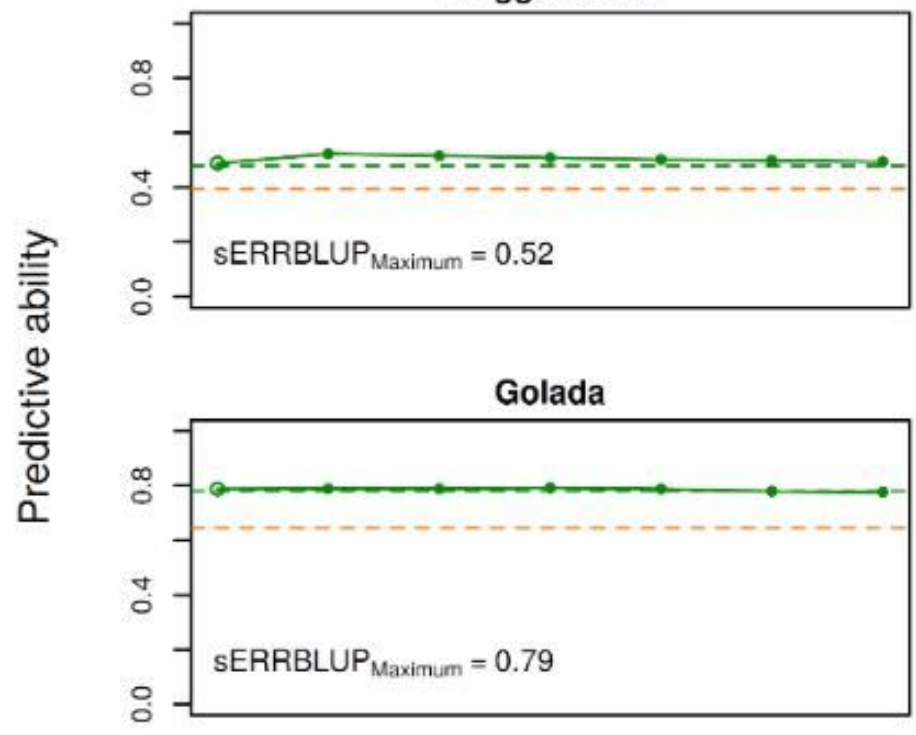

Tomeza

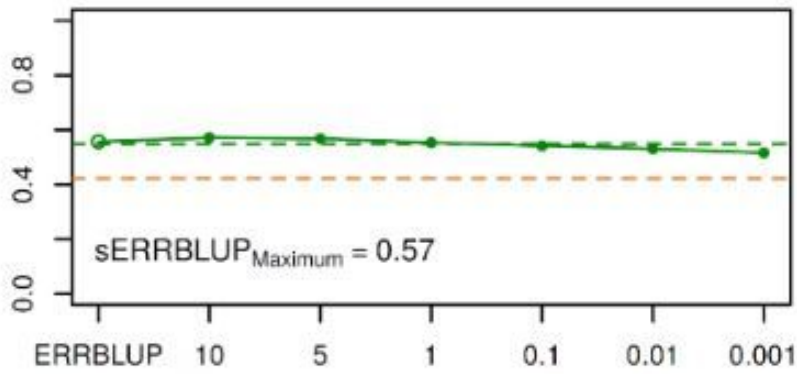

Einbeck

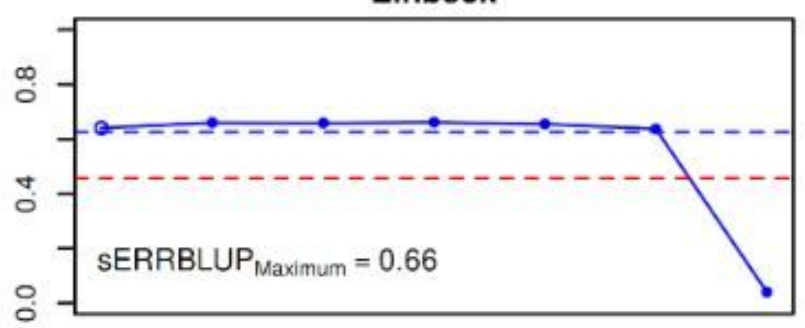

Roggenstein

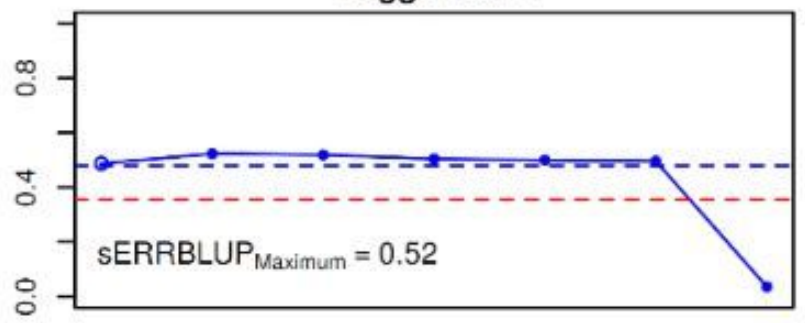

Golada

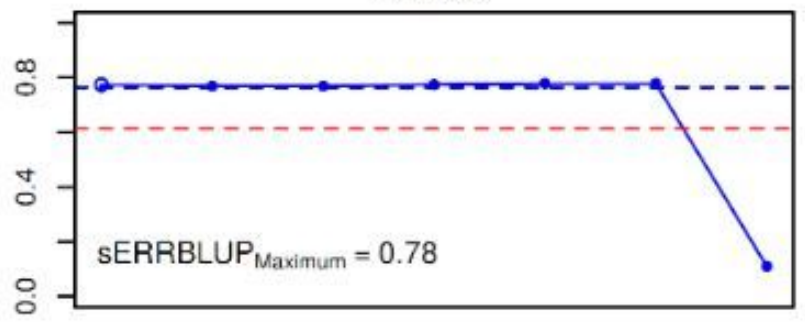

Tomeza

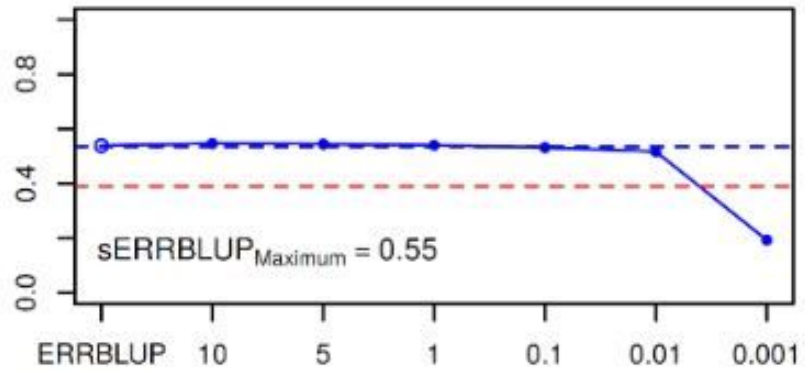

Percentage of interactions maintained in the model

\section{Figure 2}

Predictive ability for univariate GBLUP within 2018 (orange and red dashed horizontal line), bivariate GBLUP (green and blue dashed horizontal line), bivariate ERRBLUP (open circle) and bivariate sERRBLUP (filled circles and solid line) for trait PH-V4 in PE based on pruned sets of SNPs (left) and haplotype blocks (right). In each plot, the sERRBLUP maximum indicates the maximum predictive ability obtained from bivariate sERRBLUP. 

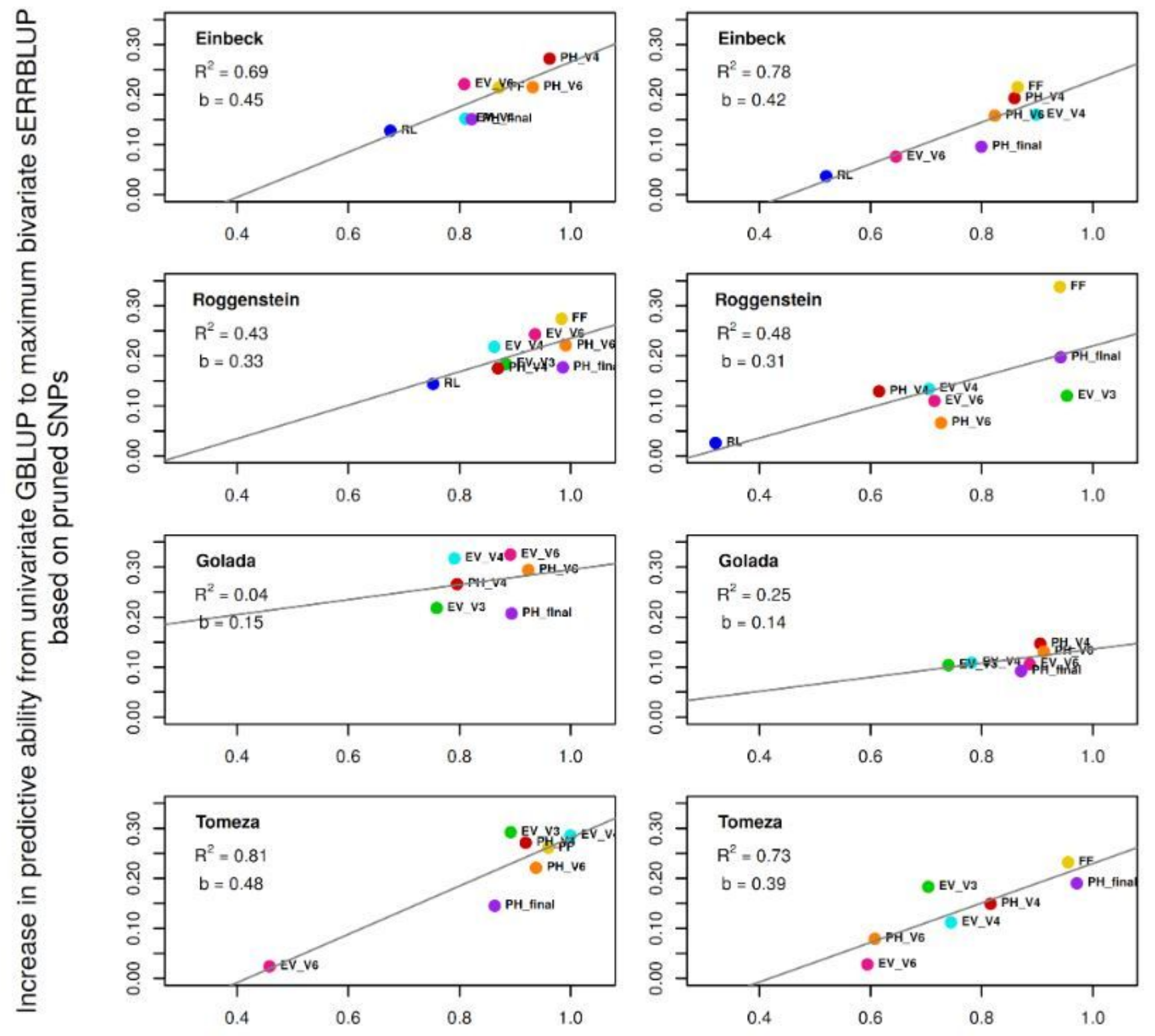

sERRBLUP genomic correlation based on pruned SNPs

\section{Figure 3}

Regression of the absolute increase in predictive ability from univariate GBLUP to maximum bivariate SERRBLUP on the respective sERRBLUP genomic correlation between 2017 and 2018 in KE (left) and in $P E$ (right) for all studied traits. In each panel, the overall linear regression line (gray solid line) with the regression coefficient $(b)$ and $R$-squared $\left(R^{\wedge} 2\right)$ are shown. 

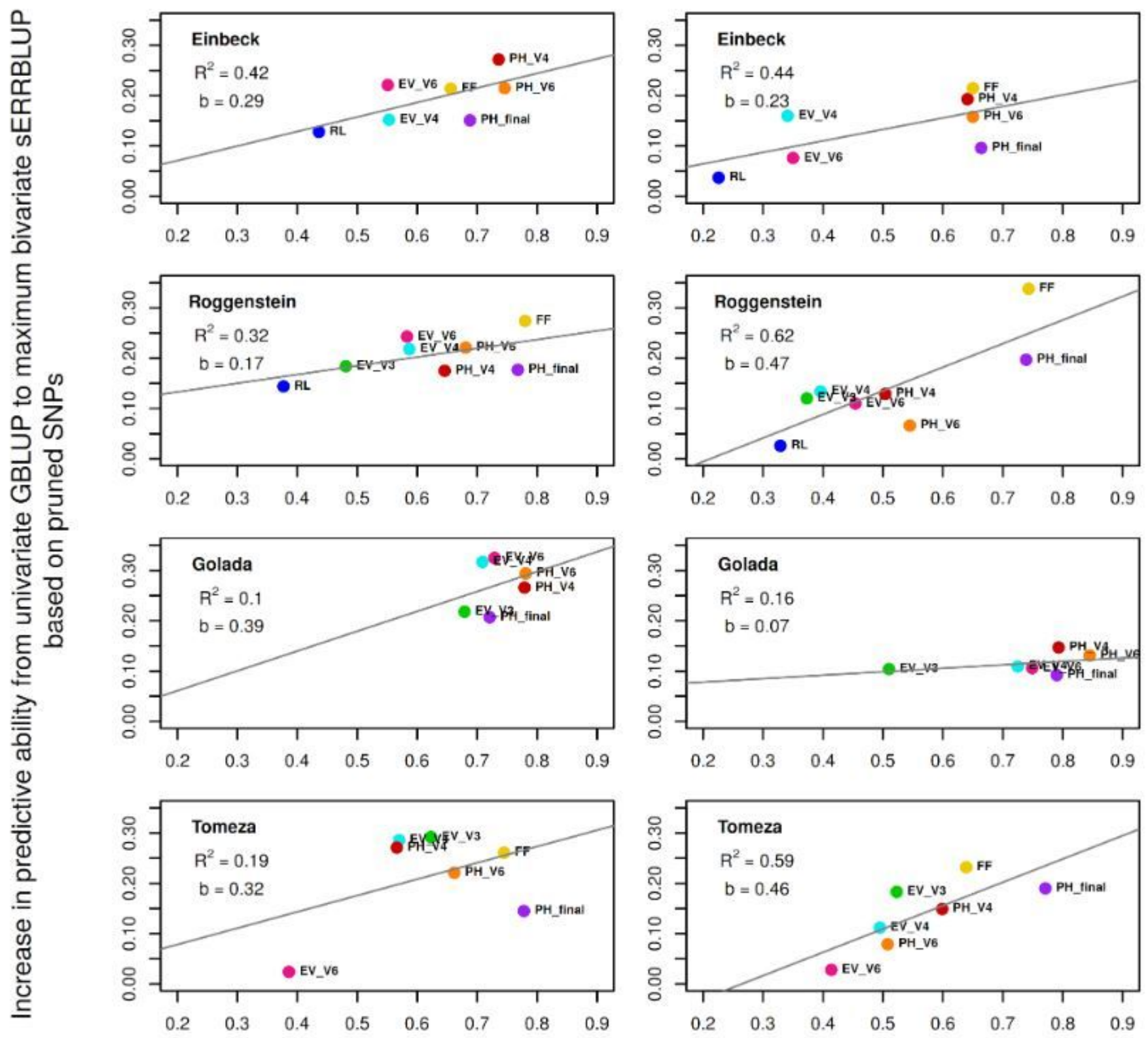

Phenotypic correlation

Figure 4

Regression of the absolute increase in predictive ability from univariate GBLUP to maximum bivariate sERRBLUP on the phenotypic correlation between 2017 and 2018 in KE (left) and in PE (right) for all studied traits. In each panel, the overall linear regression line (gray solid line) with the regression coefficient (b) and R-squared $\left(R^{\wedge} 2\right)$ are shown. 


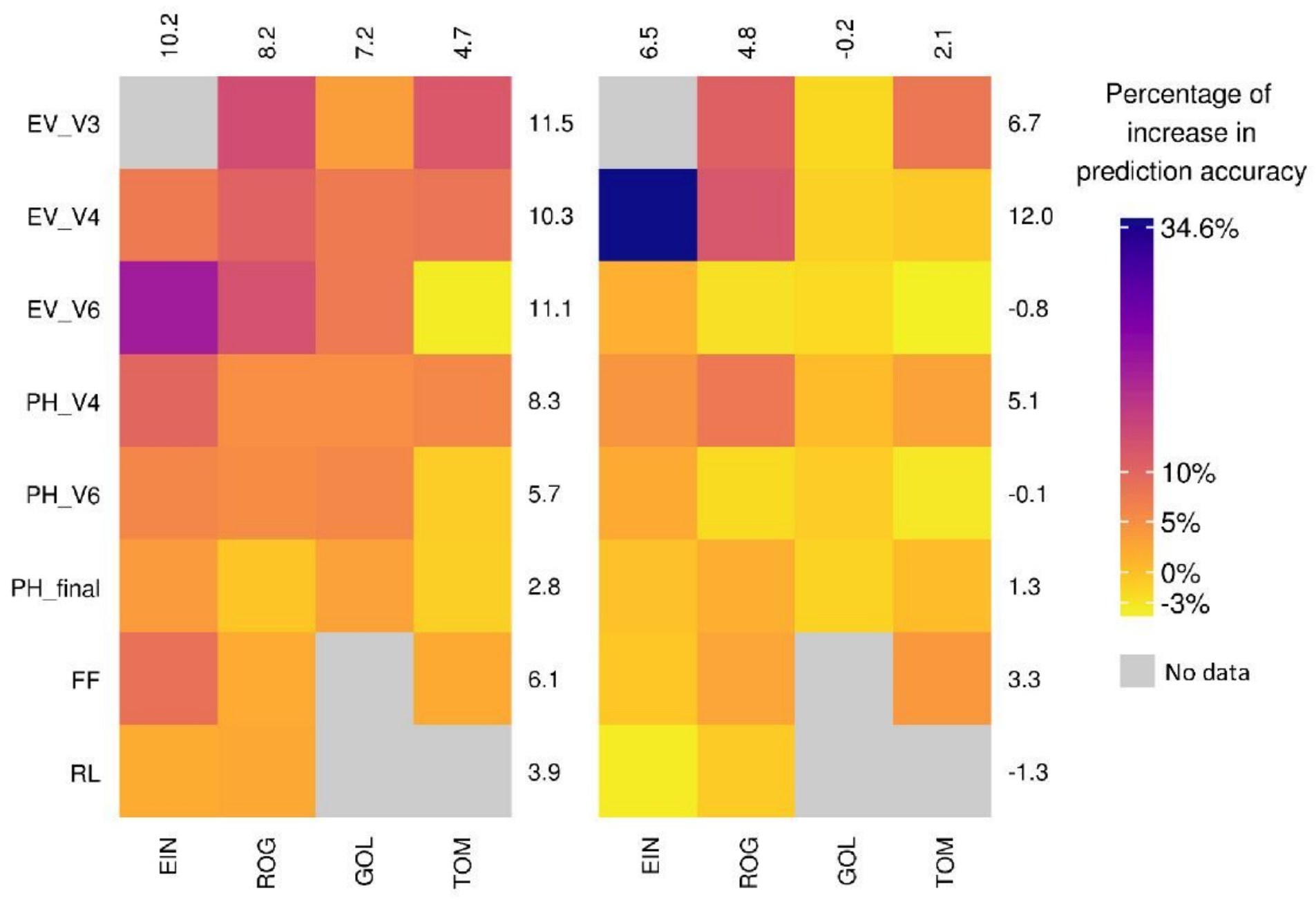

Figure 5

Percentage of change in prediction accuracy from bivariate GBLUP to the maximum prediction accuracy of bivariate sERRBLUP based on pruned set of SNPs in KE (left side plot) and in PE (right side plot). The average percentage of change in prediction accuracy for each trait and environment is displayed in all rows and columns, respectively.

\section{Supplementary Files}

This is a list of supplementary files associated with this preprint. Click to download.

- ElectronicSupplementaryMaterial.pdf 